\title{
Erratum: Layered optomagnonic structures: Time Floquet scattering-matrix approach [Phys. Rev. B 99, 144415 (2019)]
}

Petros Andreas Pantazopoulos 1 and Nikolaos Stefanou 10

(Received 28 April 2020; published 13 May 2020)

DOI: 10.1103/PhysRevB.101.209903

This is to correct Fig. 4(d) of the original paper where the numerical results suffer from convergence problems. An entire new figure, for a different set of parameters for which these problems are not encountered, is given here below. We note that the conclusions of the paper are not affected.

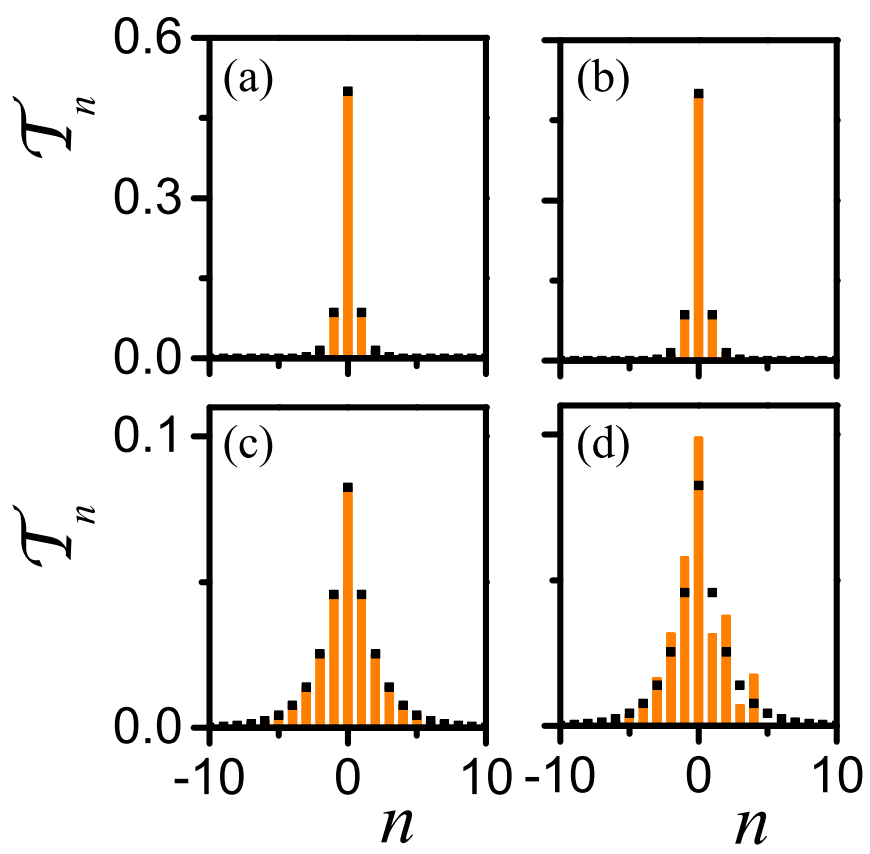

FIG. 4. Partial transmittance associated with the different plane-wave beams $\mathcal{T}_{n}$, calculated by the adiabatic approximation (points) and by the fully dynamic approach (orange bars) for different values of $\alpha_{0}$ and $\Omega / \omega$ : (a) $\alpha_{0}=1, \Omega / \omega=0.002$; (b) $\alpha_{0}=1, \Omega / \omega=0.2$; (c) $\alpha_{0}=$ $0.3, \Omega / \omega=0.002$; and (d) $\alpha_{0}=0.3, \Omega / \omega=0.2$. 